\title{
Article
}

Mycosphere

\section{Yeasts associated with Euploea butterflies}

\author{
Lin $\mathrm{WR}^{1,2}$, Wang $\mathrm{PH}^{1^{*}}$, Hsieh $\mathrm{SY}^{2}$, Tsai $\mathrm{CH}^{1}$, Hsiao $\mathrm{SC}^{1}$ \\ ${ }^{1}$ Department of Life Science, Tunghai University, No. 1727, Sec. 4, Taiwan Boulevard, Taichung 40704, Taiwan. \\ ${ }^{2}$ Bioresource Collection and Research Center (BCRC), Food Industry Research and Development Institute (FIRDI), \\ No. 331, Shih-Pin Road, Hsinchu 30062, Taiwan.
}

Lin WR, Wang PH, Hsieh SY, Tsai CH, Hsiao SC 2018 - Yeasts associated with Euploea butterflies. Mycosphere 9(1), 149-154, Doi 10.5943/mycosphere/9/1/4

\begin{abstract}
The yeasts were observed attached to the mouthparts, wings, and forelegs of migratory butterflies in Taiwan. Fifty-eight dominant yeast strains yeasts were isolated from 56 Euploea butterflies and identified by rDNA ITS and D1/D2 sequencing. The yeasts which associated with Euploea butterflies included ascomycetous yeasts, such as Aureobasidium sp., Candida chanthaburiensis, C. corydalis, Metschnikowia koreensis, Metschnikowia sp., and Debaryomyces hansenii; and basidiomycetous yeasts, such as Cryptococcus rajasthanensis, Dirkmeia churashimaensis, Filobasidium globisporum, Hannaella pagnoccae, Papiliotrema flavescens, Pseudozyma hubeiensis, $P$. tsukubaensis, Pseudozyma sp., Rhodotorula mucilaginosa, $R$. subericola, and Rhodosporidiobolus poonsookiae. The most common yeast, Candida corydalis, associated with 4 migratory Euploea species and seemed transmitted through nectar. When the butterflies feed on nectar, bask in the sun on the leaf, sip at the moisture on leaves, in puddles or wet sand and soil, they come into contact with yeasts. This is the first report about the yeast microbiome of migratory butterflies.
\end{abstract}

Key words - insect-fungus associations - insect microbiome - milkweed butterflies Saccharomycotina

\section{Introduction}

More than 400 species of butterflies have been recorded in Taiwan (Hsu 1999), and incluing four species of the tribe Danaini (i.e. Euploea tulliolus koxinga Fruhstorfer, E. sylvester swinhoei Wallace \& Moore, E. mulciber barsine Fruhstorfer and E. eunice hobsoni Butler). Species of Euploea overwinter as adults and have migration behaviour as the Monarch butterfly (Danaus plexippus) (Chao et al. 2007). From April to September, they are widely distributed in Taiwan. When air temperature decreases in winter, they migrate from north to south and overwinter from October to March in several warm and windless valleys in Lukuea, Taiwu, Laiyi and Dawu (Wang \& Emmel 1991). Their adults feed on nectars of flowers, such as Eupatorium spp., Lantana camara and some other species (Hsu 1999), while the larval feed on milkweeds.

The interaction between pollinators and flowers provides opportunities for transferring pollen grains and dispersing microorganisms. The pollinators such as bees, beetles, flies, hummingbirds, and bats have vectored numerous species of microbes including yeasts when they visited flowers (Brysch-Herzberg 2004, Lachance et al. 2005, Belisle et al. 2014, Pozo et al. 2014). Yeasts 
commonly present in nectar and utilize the sugars in the nectar (Herrera et al. 2010, Peay et al. 2011).

Butterflies are important pollinators, but only a small number of studies reported the yeasts associated with butterflies (Harrison et al. 2016, Whitaker et al. 2016). Migratory butterflies provide opportunities for long-distance pollen grains transferring, at the same time, they may harbor and transfer the nectar-specialist yeasts or transient yeasts. The objective of this study was to document the dominant yeast diversity associated with four migratory Euploea butterflies.

\section{Materials \& Methods}

We observed the yeasts on the mouthparts, wings, and forelegs of butterflies under a scanning electron microscope (SEM). Tiny pieces of mouthparts, wings or forelegs from butterflies were fixed with $2.5 \%$ glutaraldehyde at $4^{\circ} \mathrm{C}$ for $12 \mathrm{hrs}$ and then washed 3 times with $0.1 \mathrm{M}$ phosphate buffered saline for $15 \mathrm{~min}$. After this fixation process, the specimens were dehydrated in graded ethanol $(30 \%, 50 \%, 70 \%, 85 \%, 95 \%, 100 \%, 15 \mathrm{~min}$ each), and critical-point dried. Samples were coated with gold and examined with a scanning electron microscope.

The captured butterfly was put into a sterilized ziplock plastic bag with an acid YM plate (Yarrow 1998) for 15 minutes. They stood on the medium and suck it very often, their mouthparts, legs, and wings touched the medium. We released the butterflies and collected the plates. All plates were incubated at room temperature three days and checked daily for yeast growth. There were diverse yeasts in an individual butterfly, a dominant colony type of each sample was selected and purified. Fifty-eight yeast strains which belong to 17 yeast species and 9 genera were isolated from 56 individuals of 4 Euploea butterflies (4 Euploea tulliolus koxinga, 6 E. sylvester swinhoei, 30 E. mulciber barsine and $16 \mathrm{E}$. eunice hobsoni). To preserve the isolated yeasts, they were grown on $\mathrm{YM}$ agar plates for $3-7 \mathrm{~d}$ at $25^{\circ} \mathrm{C}$, transferred to broth cultures supplemented with $30 \%(\mathrm{w} / \mathrm{v})$ glycerol and stored at $-80^{\circ} \mathrm{C}$. Total DNA from the yeasts was extracted by the CTAB method (Doyle \& Doyle 1990). For sequence analyses of the yeasts, the PCR amplification of the rDNA ITS and D1/D2 region was undertaken using the universal primers ITS1/ITS4 (White et al. 1990) and F63/LR3 (Fell et al. 2000), respectively. PCR products were directly sequenced by an ABI PRISM 3730 Genetic Analyzer (PE Applied Biosystems, Foster City, CA, USA). Yeast strains were identified using nucleotide sequences. The nucleotide sequences were compared with sequences deposited in NCBI GenBank by BLAST, with the defaults settings. We assigned names to the sequences according to the following criteria: (1) sequence identity of $\geq 98.0 \% ;(2) \geq 1.0 \%$ separation from the next closest species; (3) $\geq 95.0 \%$ sequence coverage for the matching sequence; and (4) matching sequence published in a peer-reviewed journal article or submitted by the culture collection centers (CLSI 2008). Additionally, some species which were not able to identify by ITS sequences, such as Cryptococcus species, were identified to species level based on LSU sequences following the same criteria.

\section{Results}

SEM pictures show that there were typical globose or ellipsoid yeast cells attached on the mouthparts, wings, and forelegs of butterflies which collected in the field (Fig. 1). In Fig. 1, the budding yeast cells on the mouthpart and foreleg indicated that they could grow there. According to their ITS and D1/D2 sequences, the dominant yeasts which associated Euploea butterflies included Aureobasidium sp., Candida chanthaburiensis, C. corydalis, Cryptococcus rajasthanensis, Debaryomyces hansenii, Dirkmeia churashimaensis, Filobasidium globisporum, Hannaella pagnoccae, Metschnikowia koreensis, Metschnikowia sp., Papiliotrema flavescens, Pseudozyma hubeiensis, P. tsukubaensis, Pseudozyma sp., Rhodotorula mucilaginosa, R. subericola, and Rhodosporidiobolus poonsookiae (Table 1). Candida corydalis was isolated most frequently and associated with four Euploea species. There were 3, 4, 16 and 12 isolates of C. corydalis isolated as dominant from 3 Euploea tulliolus koxinga, 4 E. sylvester swinhoei, 12 E. mulciber barsine and 11 E. eunice hobsoni, respectively. Dirkmeia churashimaensis and P. hubeiensis both associated with an E. mulciber barsine and an E. eunice hobsoni. The other 14 species were found as dominant 
yeasts only on individual butterflies. Among 18 yeast species, Candida corydalis, Cryptococcus rajasthanensis, Dirkmeia churashimaensis, Pseudozyma hubeuensis, Pseudozyma tsukubaensis and Rhodosporidiobolus poonsookiae were new records to Taiwan.
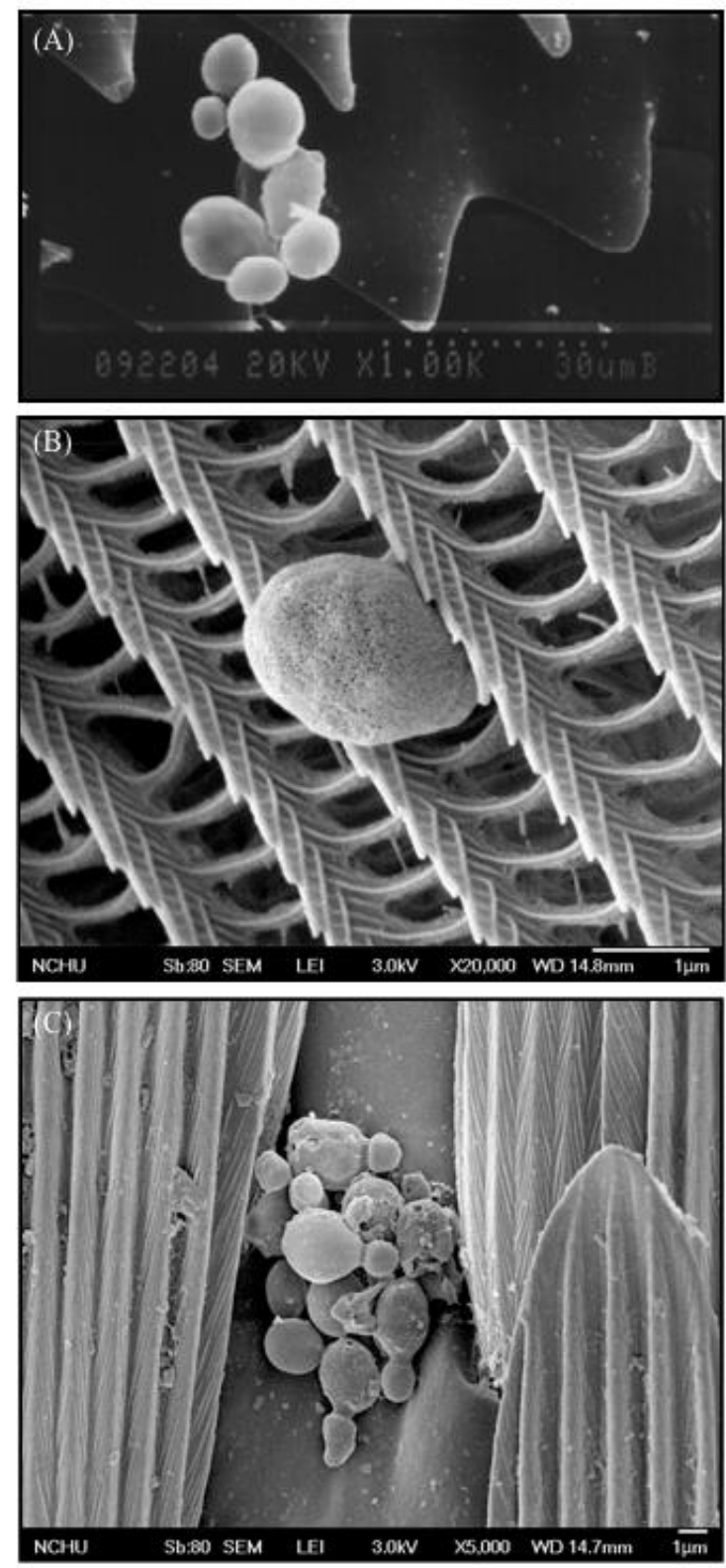

Figure 1 - The SEM pictures show that there were yeasts on the mouthparts (A) wings. (B) and (C) forelegs of butterflies which collected in the field.

\section{Discussion}

In this study, Candida and Metschnikowia were the most common yeasts associated with Euploea butterflies. They were found in highly abundance in flower nectar (Lachance et al. 2001, Brysch-Herzberg 2004, Lachance 2006, Herrera et al. 2009, Pozo et al. 2011, Belisle et al. 2012, Pozo et al. 2011) and considered as specialized nectar-dwelling yeasts (Brysch-Herzberg 2004, Lachance 2006, Herrera et al. 2010). Most of them grow fast and are able to tolerate an environment of high osmotic concentration (Lachance et al. 2001, Alvarez-Pérez \& Herrera 2013, Mittelbach et al. 2015, Mittelbach et al. 2016). Candida was the most common yeasts associated with pollinating bees (Sandhu \& Waraich 1985) and Drosophila (Chandler et al. 2012). 
Candida corydali was isolated most frequently from the 4 Euploea butterfly species and nectar of their nectariferous plants Eupatorium tashiroi Hayata and Bidens pilosa (our unpublished data). These butterflies might get the yeasts by sucking the nectar and cleaning the mouthpart by the front pair of legs, the yeasts harbored on the butterfly. The budding yeast cells on the mouthpart and foreleg of Euploea butterflies were observed by SEM demonstrated that they grew there, which indicated some yeasts may inhabit on butterfly as an ecological niche. These 4 migratory Euploea butterfly species could be the carriers to disperse yeasts.

In this study, basidiomycetes yeasts Cryptococcus, Pseudozyma, Rhodotorula, and Rhodosporidiobolus were also isolated as dominant yeast. They are primary phyllosphere colonizers (Allen et al. 2006, Last \& Price 1969, Fonseca \& Inácio 2006, Mittelbach et al. 2015) or come from soil (Fonseca \& Inácio, 2006, Yurkov et al. 2012). The butterflies may carry these transient basidiomycetous yeasts by sucking up water from leaf surface or staying at the phylloplane for foraging and basking (Mittelbach et al. 2016). They were rarely isolated in the flower nectar and have been hardly associated with nectar-foraging insects (Lachance et al. 2001, Brysch-Herzberg 2004, Pozo et al. 2011, Mittelbach et al. 2016). A next-generation sequencing study show that Malassezia restricta, $M$. globose and Rhodosporidium diobovatum associated with Lycaeides melissa adults developed from larval in the laboratory (Harrison et al. 2016). These three species belong to basidiomycetous yeasts, they persisted on the larval and leaves of larval food plant (Harrison et al. 2016). Their results indicated that butterfly can obtain basidiomycetous yeasts from phylloplane.

Our results demonstrated at least 17 dominant yeast species harbored on the mouthparts, wings, and forelegs of 4 migratory Euploea butterflies, including nectariferous ascomycetous yeasts and phyllosphere basidiomycetous yeasts. When the butterflies feed on nectar, bask in the sun on the leaf, sip at the moisture on the leaf, in puddles or wet sand and soil, they come into contact with yeasts. There are high diversity yeasts in nectar, litter, leaf surfaces, fruits, plant tissues (Fonseca \& Inácio 2006) and soil (Yurkov et al. 2012). To our knowledge, this is the first report about the yeast microbiome of migratory butterflies.

Table 1 Yeasts associated with Euploea butterflies.

\begin{tabular}{|c|c|c|c|c|c|}
\hline & \multicolumn{4}{|c|}{ Yeasts associated with Euploea butterflies } & \multirow[b]{2}{*}{ Tota } \\
\hline & $\begin{array}{c}\text { E. tulliolus } \\
\text { koxinga }\end{array}$ & $\begin{array}{c}\text { E. sylvester } \\
\text { swinhoei }\end{array}$ & $\begin{array}{c}\text { E. mulciber } \\
\text { barsine }\end{array}$ & $\begin{array}{c}\text { E. eunice } \\
\text { hobsoni }\end{array}$ & \\
\hline Aureobasidium sp. & 1 & 0 & 0 & 0 & 1 \\
\hline Candida chanthaburiensis & 0 & 1 & 0 & 0 & 1 \\
\hline Candida corydali & 3 & 4 & 16 & 12 & 35 \\
\hline Cryptococcus rajasthanensis & 0 & 0 & 1 & 0 & 1 \\
\hline Debaryomyces hansenii & 0 & 0 & 1 & 0 & 1 \\
\hline Dirkmeia churashimaensis & 0 & 0 & 1 & 1 & 2 \\
\hline Filobasidium globisporum & 0 & 0 & 1 & 0 & 1 \\
\hline Hannaella pagnoccae & 0 & 0 & 2 & 0 & 2 \\
\hline Metschnikowia koreensis & 0 & 0 & 2 & 0 & 2 \\
\hline Metschnikowia sp. & 0 & 0 & 1 & 0 & 1 \\
\hline Papiliotrema flavescens & 0 & 0 & 1 & 0 & 1 \\
\hline Pseudozyma hubeiensis & 0 & 0 & 4 & 1 & 5 \\
\hline Pseudozyma sp. & 0 & 0 & 0 & 1 & 1 \\
\hline Pseudozyma tsukubaensis & 0 & 0 & 1 & 0 & 1 \\
\hline Rhodotorula mucilaginosa & 0 & 1 & 0 & 0 & 1 \\
\hline Rhodotorula subericola & 0 & 0 & 0 & 1 & 1 \\
\hline Rhodosporidiobolus poonsookiae & 0 & 0 & 1 & 0 & 1 \\
\hline Numbers of yeast strains & 4 & 6 & 32 & 16 & 58 \\
\hline Numbers of yeast species & 2 & 3 & 12 & 5 & 17 \\
\hline
\end{tabular}




\section{Acknowledgements}

We thank Dr. Ren-Fang Chao and Jin-Yu Lu from I-Shou University, Jin-Shan Liao, RuiXiang Chen and Chih-Hao Hsu from Taiwan Purple Crow Butterfly Ecological Preservation Association, Ching-Po Cho and Yun-Shu Hsieh from Butterfly Conservation Society of Taiwan, and Yi-Ping Chang, and Hui-Lin Wu from Tunghai University for their assistance to collect butterfly.

\section{References}

Allen TW, Burpee LL, Buck JW. 2006 - Variable adhesion and diurnal population patterns of epiphytic yeasts on creeping bentgrass. Canadian Journal of Microbiology 52, 404-410.

Alvarez-Pérez S, Herrera CM. 2013 - Composition, richness and nonrandom assembly of culturable bacterial-microfungal communities in floral nectar of Mediterranean plants. FEMS Microbiology Ecology 83, 685-699.

Belisle M, Mendenhall CD, Oviedo Brenes F, Fukami T. 2014 - Temporal variation in fungal communities associated with tropical hummingbirds and nectarivorous bats. Fungal Ecology $12,44-51$.

Belisle M, Peay K, Fukami T. 2012 - Flowers as islands: spatial distribution of nectar-inhabiting microfungi among plants of Mimulus aurantiacus, a hummingbird-pollinated shrub. Microbial Ecology 63, 711-718.

Brysch-Herzberg M. 2004 - Ecology of yeasts in plant-bumblebee mutualism in Central Europe. FEMS Microbiology Ecology 50, 87-100.

Chandler JA, Eisen JA, Kopp A. 2012 - Yeast communities of diverse drosophila species: Comparison of two symbiont groups in the same hosts. Applied and Environmental Microbiology 78, 7327-7336.

Chao R-F, Hsu C-J, Chen T-Y, Yang P-S. 2007 - Overwintering ecology of Danaine butterflies in the Dawu area, Taitung County, Southeastern Taiwan. Formosan Entomologist 27, 17-30.

CLSI. 2008 - Interpretive criteria for identification of bacteria and fungi by DNA target sequencing; approved guideline. Clinical and Laboratory Standards Institute, Wayne, PA.

Doyle J, Doyle JL. 1990 - Isolation of plant DNA from fresh tissue. Focus 12, 13-15.

Fell JW, Boekhout T, Fonseca A, Scorzetti G, Statzell-Tallman A. 2000 - Biodiversity and systematics of basidiomycetous yeasts as determined by large-subunit rDNA D1/D2 domain sequence analysis. International Journal of Systematic and Evolutionary Microbiology 50, 1351-1371.

Fonseca Á, Inácio J. 2006 - Phylloplane yeasts. In: Péter DG, Rosa PC (eds), Biodiversity and Ecophysiology of Yeasts. Springer, Berlin Heidelberg, pp 263-301.

Harrison JG, Urruty DM, Forister ML. 2016 - An exploration of the fungal assemblage in each life history stage of the butterfly, Lycaeides melissa (Lycaenidae), as well as its host plant Astragalus canadensis (Fabaceae). Fungal Ecology 22, 10-16.

Herrera CM, Canto A, Pozo MI, Bazaga P. 2010 - Inhospitable sweetness: nectar filtering of pollinator-borne inocula leads to impoverished, phylogenetically clustered yeast communities. Proceedings of the Royal Society of London. Series B, Biological sciences 277, 747-754.

Herrera CM, de Vega C, Canto A, Pozo MJ. 2009 - Yeasts in floral nectar: a quantitative survey. Annals of Botany 103, 1415-1423.

Hsu YF. 1999 - Butterflies of Taiwan, Vol. 1. National Fonghuanggu Bird Park, Nantou, Taiwan.

Lachance M-A, Ewing CP, Bowles JM, Starmer WT. 2005 - Metschnikowia hamakuensis sp. nov., Metschnikowia kamakouana sp. nov. and Metschnikowia mauinuiana sp. nov., three endemic yeasts from Hawaiian nitidulid beetles. International Journal of Systematic and Evolutionary Microbiology 55, 1369-1377.

Lachance M-A, Starmer WT, Rosa CA, Bowles JM et al. 2001 - Biogeography of the yeasts of ephemeral flowers and their insects. FEMS Yeast Research 1, 1-8. 
Lachance M-A. 2006 - Yeast Biodiversity: How Many and How Much? In: Péter DG, Rosa PC (eds), Biodiversity and Ecophysiology of Yeasts. Springer, Berlin Heidelberg, pp 1-9.

Last FT, Price D. 1969 - Yeasts Associated with Living Plants and Their Environs. In: Rose A, Harrison J (eds), Yeasts, Vol 1. Academic, London, pp 183-218.

Mittelbach M, Yurkov AM, Nocentini D, Nepi M. 2015 - Nectar sugars an et ald bird visitation define a floral niche for basidiomycetous yeast on the Canary Islands. BMC Ecology 15, 2.

Mittelbach M, Yurkov AM, Stoll R, Begerow D. 2016 - Inoculation order of nectar-borne yeasts opens a door for transient species and changes nectar rewarded to pollinators. Fungal Ecology 22, 90-97.

Peay KG, Belisle M, Fukami T. 2011 - Phylogenetic relatedness predicts priority effects in nectar yeast communities. Proceedings of the Royal Society of London B: Biological Sciences, rspb20111230.

Pozo MI, Herrera CM, Alonso C. 2014 - Spatial and temporal distribution patterns of nectarinhabiting yeasts: how different floral microenvironments arise in winter-blooming Helleborus foetidus. Fungal Ecology 11, 173-180.

Pozo MI, Herrera CM, Bazaga P. 2011 - Species richness of yeast communities in floral nectar of southern Spanish plants. Microbial Ecology 61, 82-91.

Sandhu DK, Waraich MK. 1985 - Yeasts associated with pollinating bees and flower nectar. Microbial Ecology 11, 51-58.

Wang HY, Emmel TC. 1991 - Migration and overwintering aggregations of nine danaine butterfly species in Taiwan (Nymphalidae). Journal of the Lepidopterists' Society 44, 216-228.

Whitaker MRL, Salzman S, Sanders J, Kaltenpoth M, Pierce NE. 2016 - Microbial communities of Lycaenid butterflies do not correlate with larval diet. Frontiers in Microbiology 7, 1920.

White TJ, Bruns T, Lee SJ, Taylor JW. 1990 - Amplification and direct sequencing of fungal ribosomal RNA genes for phylogenetics. In: Innis MA, Gelfand DH, Sninsky JJ, White TJ (eds), PCR protocols: a guide to methods and applications. Academic Press, San Diego, pp 315-322; http://dx.doi.org/ 10.1016/b978-0-12-372180-8.50042-1.

Yarrow D. 1998 - Chapter 11 - Methods for the isolation, maintenance and identification of yeasts. In: Kurtzman CP, Fell JW (eds), The Yeasts (Fourth Edition). Elsevier, Amsterdam, pp 77100.

Yurkov AM, Kemler M, Begerow D. 2012 - Assessment of yeast diversity in soils under different management regimes. Fungal Ecology 5, 24-35. 